\title{
Role of Early Evaluation for Clonal Heterogeneity in Acute Myeloid Leukemia
}

\author{
Matthew Stein ${ }^{1}$, Syed Sameer Nasir ${ }^{2,3^{*}}$, Brennan Palazola ${ }^{1}$, Melissa Crawley ${ }^{2,3}$, Mike G Martin ${ }^{2,3}$
}

${ }^{1}$ Department of Medicine, University of Tennessee Health Science Center, Memphis, TN

${ }^{2}$ Division of Hematology \& Oncology, Department of Medicine, University of Tennessee Health Science Center, Memphis, TN ${ }^{3}$ The West Cancer Center, Memphis, TN

*Corresponding author: Syed Sameer Nasir, MD, The University of Tennessee Health Science Center/West Cancer Center, 1588 Union Ave, c/o Dr. Mike G. Martin, Memphis, TN 38104, Fax: (901) 322-0257; E-mail: snasir@westclinic.com

Citation: Nasir, S.S., et al. Role of Early Evaluation for Clonal Heterogeneity in Acute Myeloid Leukemia. (2017) Int J Hematol Ther 3(1): 1- 2.
Received Date: January 27, 2017

Accepted Date: March 17, 2017

Published Date: March 22, 2017

DOI: $10.15436 / 2381-1404.17 .020$

\section{Case Report}

A 67-year-old Caucasian male presented with a chief complaint of shortness of breath and fatigue. Initial laboratory evaluation revealed hyperleukocytosis with WBC count of $76,000 / \mathrm{mcL}$ and $83 \%$ blasts. He immediately received leukapheresis for respiratory symptoms. Bone marrow biopsy and peripheral blood flow cytometry confirmed a diagnosis of acute myeloid leukemia (AML) with normal cytogenetics. Fluorescence in-situ hybridization (FISH) study of the bone marrow was negative for $t(8 ; 21), t(15 ; 17)$, inv(16) and abnormalities of Mixed Lineage Leukemia. Mutation analysis by PCR (Quest Diagnostics, AML Prognostic Panel) showed mutations in nucleophosmin (NPM1) gene and internal tandem duplication in the FMS-like Tyrosine Kinase 3 gene (FLT3-ITD) but wild-type for CCAAT/enhancer binding protein $\alpha$ gene (CEBPA).

The patient received induction chemotherapy with cytarabine and idarubicin $(7+3)$. Day-14bone marrow biopsy showed a hypocellular marrow with no morphologic evidence of residual AML. Cytogenetic analysis was unsuccessful due to lack of metaphases. Repeat mutational analysis was also completed on the day-14 bone marrow sample and was found to be positive for FLT3-ITD but negative for NPM1. The day-30 bone marrow biopsy showed evidence of residual AML with normal cytogenetics and $67 \%$ blasts. He subsequently underwent salvage chemotherapy with cladribine, cytarabine and filgrastrim (CLAG regimen). Despite salvage chemotherapy his follow-up bone marrow continued to have high-level residual disease with $78 \%$ blasts and persistent FLT3-ITD positivity. He was then started on sorafenib and azacitidine and following one cycle entered a complete remission. Repeat molecular studies at the time of morphologic remission showed continued presence of the FLT3-ITD. At the time of writing this report, the patient has completed 3 cycles of sorafenib and azacitidine therapy and remains in remission and is proceeding with an allogeneic stem cell transplant.

Pretreatment characterization of adult patients with AML for risk of relapse is based upon cytogenetic analysis and molecular profiling that can distinguish broad subgroups of patients with relatively favorable, intermediate, or unfavorable prognosis ${ }^{[1]}$. However, approximately $50 \%$ of patients experience relapse after achieving complete remission with initial induction chemotherapy ${ }^{[2]}$. This is believed to be due in part to clonal heterogeneity ${ }^{[3]}$, suggesting the need for well-timed strategies to assess molecular response and optimize therapy. Recently, Klco., et al showed that detection of persistent AML-associated mutations in day-30 remission samples was associated with a significantly increased risk of relapse, and reduced overall survival. This was corroborated by Ivey., et al, showing presence of NPM1-mutated transcripts in peripheral blood after second chemotherapy cycle was significantly associated with higher risk of relapse at 3 years $^{[4]}$. These observations suggested that the presence of minimal residual disease was a better predictor of relapse. However, we did not find data on prognostic value of 
mutational studies on day-14 bone marrow samples after initial induction therapy. Here, we report a case where evaluation of day-14 bone marrow for AML-associated mutations was helpful in identifying clonal heterogeneity and following response to induction chemotherapy. Our patient had refractory FLT3ITD AML with evidence of clonal heterogeneity as he cleared NPM1 positive clones with persistence of FLT3-ITD clones on day 14 bone marrow, which were refractory to chemotherapy but responded to sorafenib and azacitidine therapy ${ }^{[5]}$. This finding raises the question of the prognostic implication of early cytogenetic and molecular testing during induction therapy for AML to validate initial risk stratification and in making timely treatment decisions regarding further therapy as well as the application of targeted therapies.

Conflict of Interest: The authors declare no competing financial interests.

\section{References:}

1. El Rassi, F., Arellano, M. Update on optimal management of acute myeloid leukemia. (2013) Clin Med Insights Oncol 7: 181-197.

Pubmed I Others

2. Klco, J.M., Miller, C.A., Griffith, M., et al. Association between mutation clearance after induction therapy and outcomes in acute myeloid leukemia. (2015) JAMA 314(8): 811-822.

Pubmed I Crossref I Others

3. Ding, L., Ley, T.J., Larson, D.E., et al. Clonal evolution in relapsed acute myeloid leukemia revealed by whole-genome sequencing. (2012) Nature 481(7382): 506-510.

Pubmed । Crossref । Others

4. Ivey, A, Hills, RK, Simpson, MA, et al. Assessment of Minimal Residual Disease in Standard-Risk AML. (2016) N Engl J Med 375(6): e9. Pubmed I Crossref I Others

5. Ravandi, F., Alattar, M.L., Grunwald, M.R., et al. Phase 2 study of azacytidine plus sorafenib in patients with acute myeloid leukemia and FLT-3 internal tandem duplication mutation. (2013) Blood 121(23): 4655-4662.

Pubmed I Crossref I Others
Ommega Online Publisher International Journal of Hematology \& Therapy Short Title : Int J Hematol Ther
ISSN: 2381-1404

E-mail : hematology.therapy@ommegaonline.org website: www.ommegaonline.org 